\title{
Clinical characteristics and outcomes of critically III patients with COVID-19 in Northeast Ohio: low mortality and length of stay
}

\author{
Francois Abi Fadel ${ }^{1,2}$, Mohammed Al-Jaghbeer ${ }^{1,2}$, Sany Kumar ${ }^{3}$, Lori Griffiths ${ }^{4}$, Xiaofeng Wang ${ }^{5}$, \\ Xiaozhen Han $^{5}$, Robert Burton ${ }^{6}$ \\ ${ }^{1}$ Respiratory Institute, Cleveland Clinic, Cleveland, $\mathrm{OH}_{;}^{2} \mathrm{Cleveland}$ Clinic Lerner College of Medicine of Case Western Reserve University, $\mathrm{Cleveland}, \mathrm{OH}$; \\ ${ }^{3}$ Fairview Hospital, ${ }^{4}$ Quality Data Registries, ${ }^{5}$ Quantitative Health Sciences, and ${ }^{6}$ Business Intelligence, Cleveland Clinic, Cleveland, OH, USA
}

Background: Published coronavirus disease 2019 (COVID-19) reports suggest higher mortality with increasing age and comorbidities. Our study describes the clinical characteristics and outcomes for all intensive care unit (ICU) patients admitted across the Cleveland Clinic enterprise, a 10-hospital health care system in Northeast Ohio, serving more than 2.7 million people. Methods: We analyzed the quality data registry for clinical characteristics and outcomes of all COVID-19-confirmed ICU admissions. Differences in outcomes from other health care systems and published cohorts from other parts of the world were delineated.

Results: Across our health care system, 495 COVID-19 patients were admitted from March 15 to June 1, 2020. Mean patient age was 67.3 years, 206 (41.6\%) were females, and 289 (58.4\%) were males. Mean Acute Physiology Score was 45.3, and mean Acute Physiology and Chronic Health Evaluation III score was 60.5. In total, 215 patients (43.3\%) were intubated for a mean duration of 9.2 days. Mean ICU and hospital length of stay were 7.4 and 13.9 days, respectively, while mean ICU and hospital mortality rates were $18.4 \%$ and $23.8 \%$.

Conclusions: Our health care system cohort is the fourth largest to be reported. Lower ICU and hospital mortality and length of stay were seen compared to most other published reports. Better preparedness and state-level control of the surge in COVID-19 infections are likely the reasons for these better outcomes. Future research is needed to further delineate differences in mortality and length of stay across health care systems and over time.

Key words: cohort; COVID-19; critical illness; hospital mortality; intensive care unit; mechanical ventilation; pandemic

\section{INTRODUCTION}

Coronavirus disease 2019 (COVID-19) has spread across the globe, causing more than a half million deaths to date [1]. The first COVID-19 death in Ohio was reported on March 1, 2020, and it has affected more than 89,626 people up to July 31 , with more than 10,678 hospitalizations and 3,442 deaths across the state [2]. Studies from different countries pointed to higher mortality with increasing age and comorbidities $[3,4]$. Prior published results focused on overall hospitalized patients [3,4] or critically ill patients [5-13]. Our current study describes

\section{Original Article}

Received: August 5, 2020

Revised: September 11, 2020

Accepted: September 28, 2020

Corresponding author

Francois Abi Fadel

Respiratory Institute, Cleveland

Clinic, 9500 Euclid Ave, Cleveland,

$\mathrm{OH} 44195$, USA

Tel: +1-2164067743

Fax: +1-4408783075

E-mail: abifadf@ccf.org

Copyright () 2020 The Korean Society of Critical Care Medicine

This is an Open Access article distributed under the terms of Creative Attributions Non-Commercial License (https:// creativecommons.org/li-censes/by-nc/4.0/) which permits unrestricted noncommercial use, distribution, and reproduction in any medium, provided the original work is properly cited. 
the clinical characteristics and outcomes of all intensive care unit (ICU) patients admitted across the Cleveland Clinic enterprise, a 10-hospital health care system in Northeast Ohio with more than $60 \%$ of the market share, serving more than 2.7 million people. Through our study, we tried to explain differences in outcomes from other health care systems and published cohorts from other parts of the world.

\section{MATERIALS AND METHODS}

We analyzed the Cleveland Clinic health care system quality data registry for clinical characteristics and outcomes for all COVID-19-confirmed ICU admissions from March 15 to June 1, 2020. Data were compiled on June 29 to allow a 28-day assessment of outcomes in all patients. Table 1 summarizes demographics, clinical characteristics, and outcomes data. Before finalizing this report, we gathered available preliminary outcome data as of July 25, 2020 from all 10 hospital ICU cohorts since March. COVID-19 diagnosis was based on a positive polymerase chain reaction of a nasopharyngeal swab. We collected demographics, clinical characteristics, laboratory values at admission, ICU admission sources, admission Acute Physiology Score (APS), Acute Physiology And Chronic Health Evaluation III (APACHE III) score, hospital and ICU length of stay (LOS), hospital and ICU mortality, and duration of mechanical ventilation. The Institutional Review Board at the Cleveland Clinic approved this study (No. 20-418) and waived the need for patient informed consent. A review of other published reports on critically ill patients with COVID-19, available from the United States, Canada, Italy, the United Kingdom, and China was performed, with a focus on differences in baseline characteristics and outcomes over time. Chisquare test was used for statistical analysis and comparisons of mortality and mechanical ventilator ratios.

\section{RESULTS}

Across our health care system ICUs, 495 patients were admitted for severe COVID-19 infection from March 15 to June 1, 2020 (Table 1). Mean age was 67.3 years, 206 (41.6\%) were females, and 289 (58.4\%) were males. The majority of patients (54.9\%) was Caucasian, and 192 (38.3\%) were African American. In addition, 176 (33.7\%) were admitted from emergency rooms, and 228 (46.1\%) transferred from medical or surgical wards to the ICU. Median BMI was $29.7 \mathrm{~kg} / \mathrm{m}^{2}, 177$ patients (35.8\%) were diabetic, and 64 (12.9\%) met the criteria for severe sepsis or septic shock. Mean APS was 45.3, and mean

\section{KEY MESSAGES}

- Better preparedness and state-level control of the surge in coronavirus disease 2019 (COVID-19) infections are the most plausible explanations for the lower mortality and length of stay in our cohort.

APACHE III score was 60.5 . There were 215 patients on mechanical ventilation (43.3\%) for a mean duration of 9.2 days. Of those 215 intubated patients, 24 underwent tracheostomy and were discharged on mechanical ventilation to a nursing home with ventilator capacity or to a long-term acute care hospital (11.16\%). Mean ICU and hospital LOS values were 7.4 and 13.9 days, respectively, and ICU and hospital mortality rates were $18.4 \%$ and $23.8 \%$. Mean C-reactive protein on admission was $13.5 \mathrm{mg} / \mathrm{dl}$, mean D-dimer was $4,163.2 \mathrm{ng} / \mathrm{ml}$, mean ferritin was 2,306.5 ng/ml, mean fibrinogen was 565.5 $\mathrm{mg} / \mathrm{dl}$, mean hemoglobin Alc was $7.9 \%$, mean interleukin-6 was $116.0 \mathrm{pg} / \mathrm{ml}$, and mean triglycerides was $210.7 \mathrm{mg} / \mathrm{dl}$ (Table 2). Our reported cohort contained no cases of catheter-associated urinary tract infection, no cases of ventilator-acquired pneumonia, and one case of fungal central line-associated blood stream infection.

\section{DISCUSSION}

A review of the literature revealed several published cohorts and case series reports on demographics, clinical characteristics, and outcomes of COVID-19 patients as early as February 2020 from Wuhan, China [4]; March 2020 from Tongji Hospital also in Wuhan, China [10], Seattle and Washington state in the United States [6,12], and from the Lombardy region in Italy [5]; May 2020 from Vancouver, Canada [8]; a 65-center cohort study in the United States published July 2020 [7]; and the United Kingdom's Intensive Care National Audit and Research Center report on critically ill COVID-19 patients as of July 31, 2020 [13].

Our cohort of 495 patients is the fourth largest to be reported. Median age was 68 years, with a predominantly male population at $58.4 \%$ and elevated cohort mean BMI of $31.7 \mathrm{~kg} / \mathrm{m}^{2}$. These results are similar to all prior reported demographics, supporting older age, male sex, and elevated BMI as risk factors for critical illness and adverse outcomes of COVID-19 infection. Diabetic patients whether type 1 or type 2 representing $35.8 \%$ of the ICU COVID-19 population is consistent with findings from Vancouver, Canada [8]; Seattle, WA [6]; and 


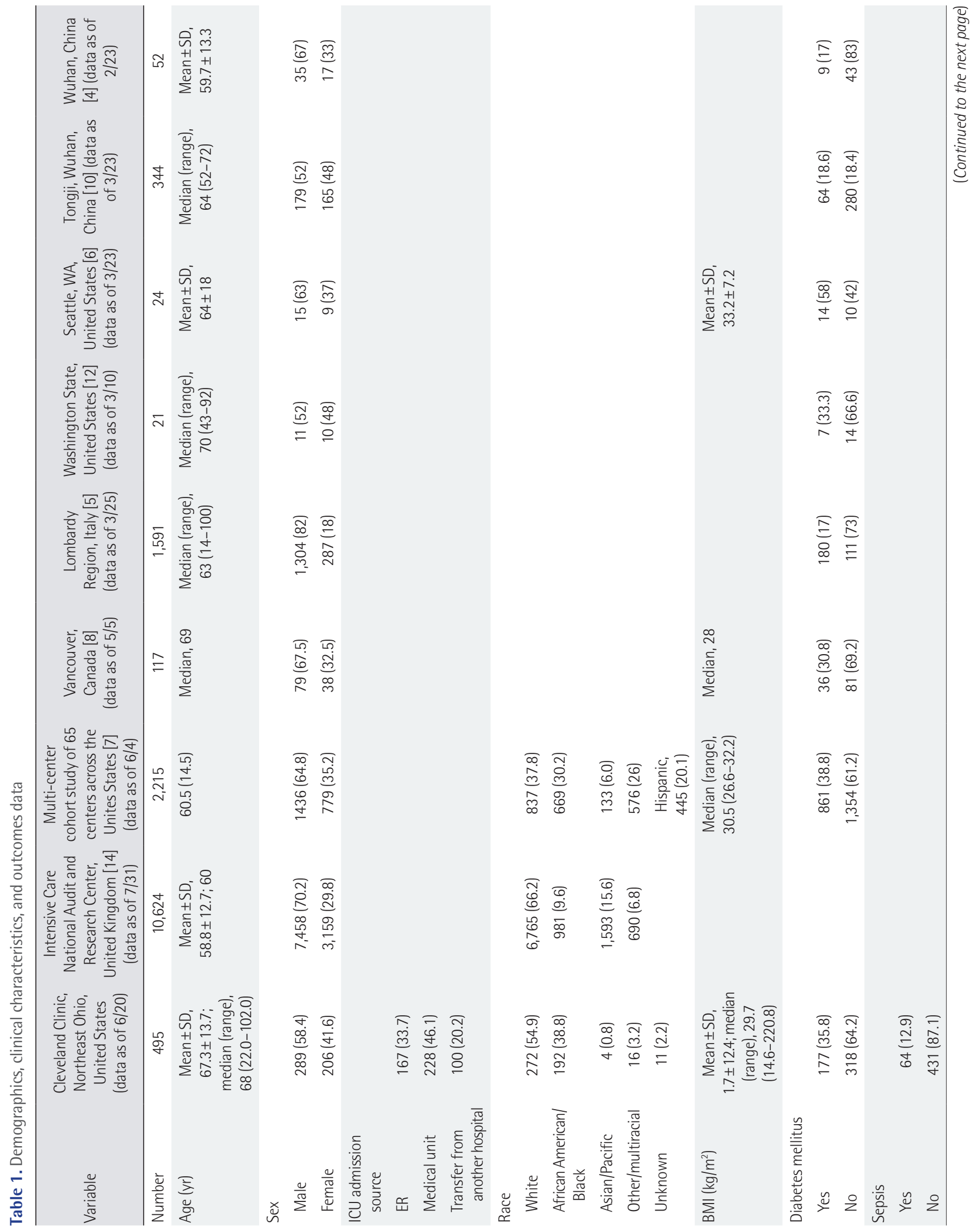




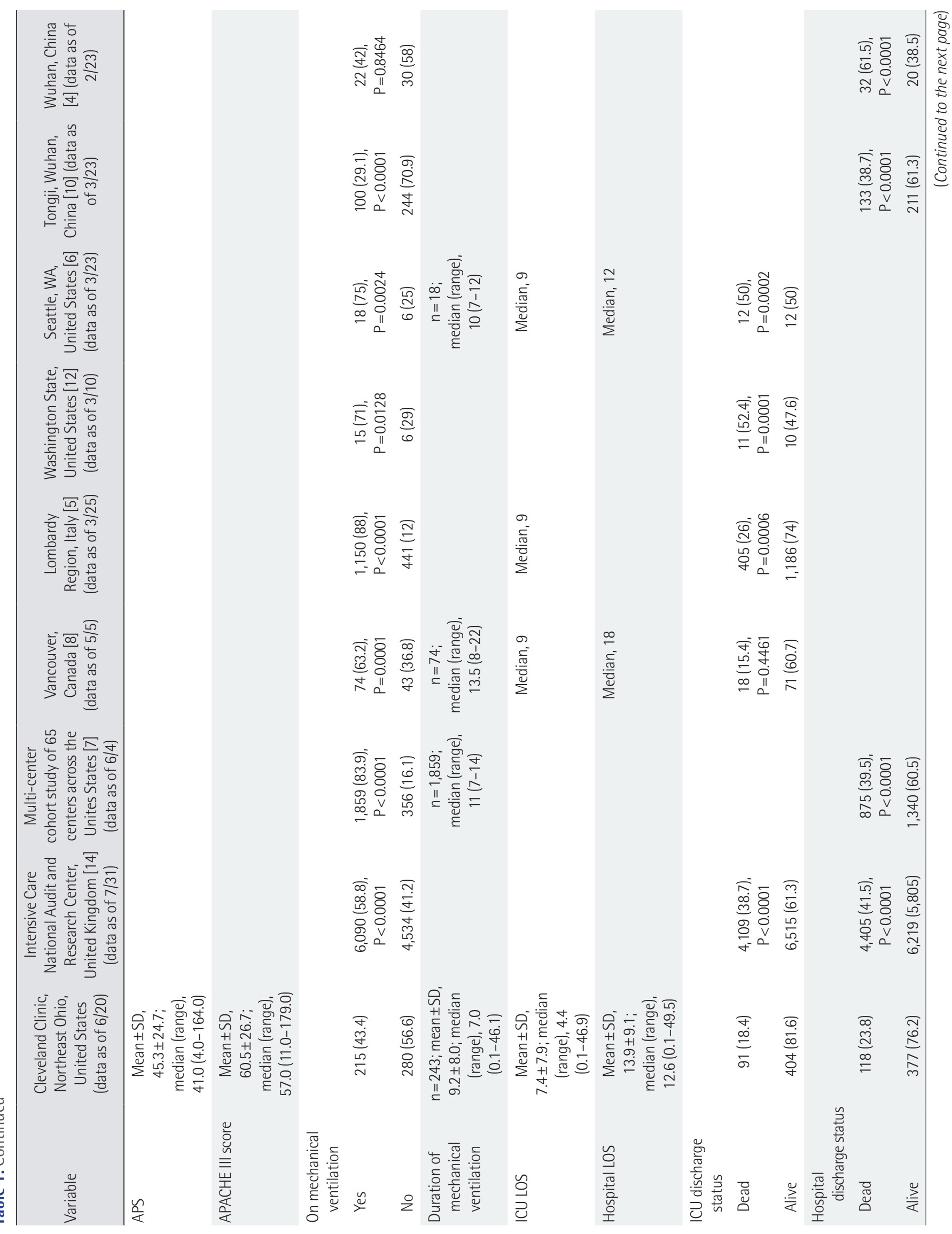




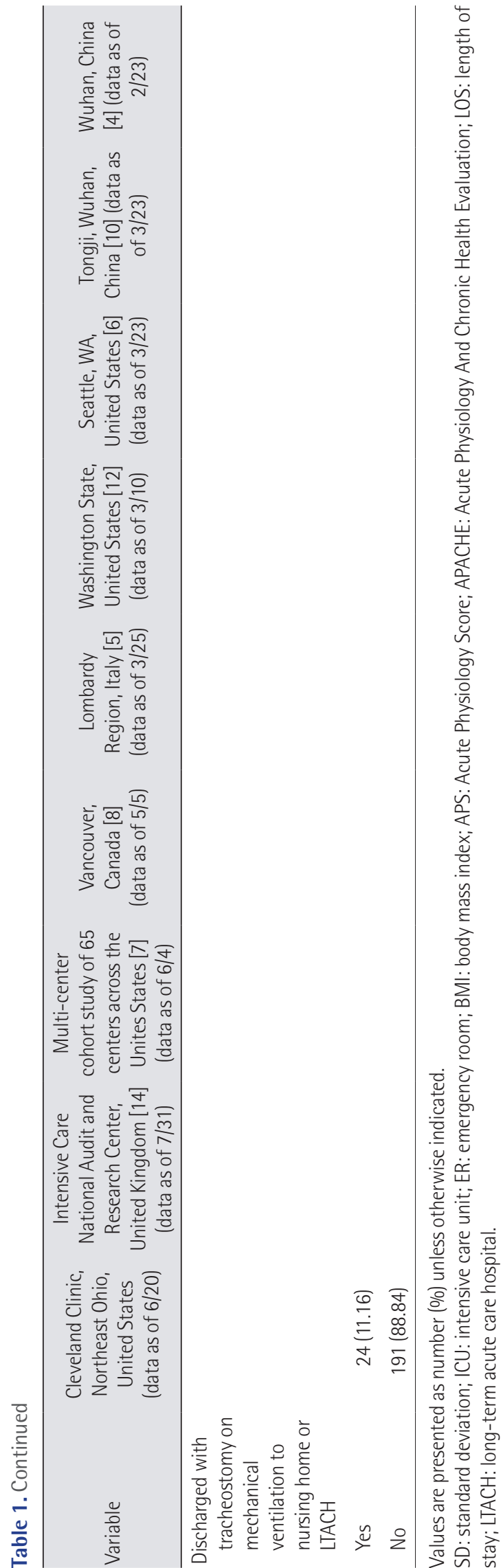

Washington state [12], as well as a multi-center cohort study across 65 centers in the United States [7]. This percentage is significantly higher than the $9.4 \%$ reported by the Center for Disease Control and Prevention Diabetes Center in Northeast Ohio, the $9.7 \%$ reported by the State of Ohio, and the $9.1 \%$ reported by the United States [15]. This confirms diabetic comorbidity to be associated with adverse outcomes including critical illness and ICU admission in COVID-19 patients.

The invasive mechanical ventilation rate in our cohort was $43.4 \%$, similar to that reported from Wuhan, China $[4,10]$. However, this result was significantly lower than all other reports of mechanical ventilator usage in critically ill patients: $63.2 \%$ in Vancouver, Canada and up to $83.9 \%$ in a multi-center cohort study of 65 centers in the United States $[5-8,12,13]$ (Table 1). Our reported ICU mortality of $18.4 \%$ and hospital mortality of $23.8 \%$ were significantly lower than those of most other cohorts except that from Vancouver, Canada [8] (Table 1). In addition, our median ICU LOS of 4.4 days was significantly shorter than that of 9 days from Lombardy, Vancouver, and Seattle $[5,8,12]$ (Table 1). When an updated outcome summary from our health care system cohort was requested on $\mathrm{Au}$ gust 7, ICU mortality had decreased from $18.4 \%$ to $17 \%$ and LOS from 7.4 to 6.6 days, while hospital mortality and LOS decreased from $23.8 \%$ to $21 \%$ and from 13.9 to 12.33 days, respectively, compared to the outcome data reported at the end of June.

A recent systematic review and meta-analysis of ICU mortality in COVID-19 patients reported a $41.6 \%$ mortality rate across all studies, with no significant effect of geographical location. However, a meta-regression by month of publication revealed a significant reduction in ICU mortality over time [11]. All studies reported an ICU COVID-19 mortality higher than the $4.4 \%$ [14] to $21.4 \%$ [13] mortality reported for those with non-COVID-19 viral and community-acquired pneumonia, and all were higher than our cohort ICU mortality of $18.4 \%$.

All data published or reported, including ours, were preliminary and incomplete, representing up to 28 days post ICU admission. Consequently, all reported morality and LOS are likely underestimated. Although unable to compare admission acuity across cohorts and case series as APS and APACHE III scores were rarely reported, several factors can explain our lower mortality and LOS. First, the state of Ohio enacted early social distancing measures, school closures, and a "stay at home" order prevented a surge in COVID-19 infection and in ICU and hospital bed utilization. These measures allowed proper time for all health care systems to prepare, and none was overwhelmed with COVID-19 patients as was seen in 
Table 2. Laboratory test results on admission of COVID-19 patients admitted to an intensive care unit

\begin{tabular}{|c|c|}
\hline Variable & Value \\
\hline \multicolumn{2}{|l|}{ CRP (mg/dl) } \\
\hline Number & 386 \\
\hline Mean \pm SD & $13.5 \pm 9.2$ \\
\hline Median & 12.2 \\
\hline 01,03 & $5.9,18.8$ \\
\hline Range & $0.1-48.3$ \\
\hline \multicolumn{2}{|c|}{ D-dimer (ng/ml) } \\
\hline Number & 330 \\
\hline Mean $\pm S D$ & $4,163.2 \pm 7,648.1$ \\
\hline Median & $1,520.0$ \\
\hline 01,03 & $850.0,3,340.0$ \\
\hline Range & $3.9-35,200.0$ \\
\hline \multicolumn{2}{|c|}{ Ferritin (ng/ml) } \\
\hline Number & 381 \\
\hline Mean $\pm S D$ & $2,306.5 \pm 9,306.1$ \\
\hline Median & 843.6 \\
\hline 01,03 & $447.0,1,633.0$ \\
\hline Range & $30.9-100,000.0$ \\
\hline \multicolumn{2}{|c|}{ Fibrinogen (mg/dl) } \\
\hline Number & 213 \\
\hline Mean $\pm S D$ & $565.5 \pm 179.5$ \\
\hline Median & 563.0 \\
\hline 01, 03 & $437.0,703.0$ \\
\hline Range & $86.0-860.0$ \\
\hline \multicolumn{2}{|l|}{ HbA1c (\%) } \\
\hline Number & 69 \\
\hline Mean \pm SD & $7.9 \pm 2.5$ \\
\hline Median & 7.1 \\
\hline 01,03 & $6.1,8.7$ \\
\hline Range & $5.0-14.4$ \\
\hline \multicolumn{2}{|c|}{ Interleukin-6 (pg/ml) } \\
\hline Number & 205 \\
\hline Mean $\pm S D$ & $116.0 \pm 204.8$ \\
\hline Median & 49.0 \\
\hline 01,03 & $14.0,120.5$ \\
\hline Range & $2.2-1,602.7$ \\
\hline \multicolumn{2}{|l|}{ Troponin T } \\
\hline Number & 260 \\
\hline Mean $\pm S D$ & $0.1 \pm 0.2$ \\
\hline Median & 0 \\
\hline 01,03 & $0.0,0.0$ \\
\hline Range & $0.0-2.0$ \\
\hline
\end{tabular}

Table 2. Continued

\begin{tabular}{lc}
\hline Variable & Value \\
\hline Triglycerides $(\mathrm{mg} / \mathrm{dl})$ & \\
Number & 321 \\
Mean \pm SD & $210.7 \pm 379.0$ \\
Median & 137 \\
Q1, 03 & $103.0,203.0$ \\
Range & $9.0-4,425.0$ \\
\hline
\end{tabular}

COVID-19: coronavirus disease 2019; CRP: C-reactive protein; SD: standard deviation; HbA1c: hemoglobin A1c.

other states such as New York and other countries such as Italy. In addition, we found a significantly lower rate of mechanical ventilation of $43.4 \%$ compared to most other cohorts and a lower median 7.0-day duration of mechanical ventilation compared to the 10 to 13.5 days reported in other studies $[6,7,8]$. Our planning and strict protocol-based best practice daily spontaneous breathing trial and sedation weaning also likely contributed to the result differences. Finally, as mentioned above, the date of data collection in June could have played a role in the observed reduction in ICU mortality since the start of the pandemic [11]. The main limitations of our study are the retrospective nature and use of data from a single health care system, which might restrict generalizability. However, this single system comprised 10 hospitals across Northeast Ohio, serving 2.7 million people.

Our analysis of outcome data from more than 495 ICU patients with a 28-day follow-up is the fourth largest to be reported. Lower ICU and hospital LOS and mortality were noted compared to most other published cohorts and case series across the globe. Better preparedness and state-level control of preventive measures seem to be the most plausible explanations for the lower mortality and LOS. Future study should determine if the lower rate of mechanical ventilation use and shorter duration on mechanical ventilation played a role.

Future research is needed to further explain the higher mortality of COVID-19 viral pneumonia compared to other viral and community-acquired pneumonias and to explain differences in mortality and LOS at national and international levels across health care systems and over time.

\section{CONFLICT OF INTEREST}

No potential conflict of interest relevant to this article was reported. 


\section{AUTHOR CONTRIBUTIONS}

Conceptualization: FAF. Data curation: FAF, MAJ, SK, LG, RB. Formal analysis: FAF, SK, XW, XH. Methodology: FAF. Project administration: FAF. Visualization: FAF. Writing-original draft: FAF, SK. Writing-review \& editing: FAF, MAJ.

\section{REFERENCES}

1. World Health Organization. WHO coronavirus disease (COVID-19) dashboard [Internet]. Geneva: World Health Organization; 2020 [cited 2020 Jul 31]. Available from: https://covid19.who.int.

2. Ohio Department of Health COVID-19 Dashboard [Internet]. 2020 [cited 2020 Jul 31]. Available from: https://coronavirus. ohio.gov/wps/portal/gov/covid-19/dashboards/overview.

3. Richardson S, Hirsch JS, Narasimhan M, Crawford JM, McGinn T, Davidson KW, et al. Presenting characteristics, comorbidities, and outcomes among 5700 patients hospitalized with COVID-19 in the New York city area. JAMA 2020;323:2052-9.

4. Zhou F, Yu T, Du R, Fan G, Liu Y, Liu Z, et al. Clinical course and risk factors for mortality of adult inpatients with COVID-19 in Wuhan, China: a retrospective cohort study. Lancet 2020;395:1054-62.

5. Grasselli G, Zangrillo A, Zanella A, Antonelli M, Cabrini L, Castelli A, et al. Baseline characteristics and outcomes of 1591 patients infected with SARS-CoV-2 admitted to ICUs of the Lombardy region, Italy. JAMA 2020;323:1574-81.

6. Bhatraju PK, Ghassemieh BJ, Nichols M, Kim R, Jerome KR, Nalla AK, et al. Covid-19 in critically ill patients in the Seattle region: case series. N Engl J Med 2020;382:2012-22.

7. Gupta S, Hayek SS, Wang W, Chan L, Mathews KS, Melamed ML, et al. Factors associated with death in critically ill patients
With coronavirus disease 2019 in the US. JAMA Intern Med 2020 Jul 15 [Epub]. https://doi.org/10.1001/jamainternmed. 2020.3596

8. Mitra AR, Fergusson NA, Lloyd-Smith E, Wormsbecker A, Foster D, Karpov A, et al. Baseline characteristics and outcomes of patients with COVID-19 admitted to intensive care units in Vancouver, Canada: a case series. CMAJ 2020;192:E694-701.

9. Yang X, Yu Y, Xu J, Shu H, Xia J, Liu H, et al. Clinical course and outcomes of critically ill patients with SARS-CoV-2 pneumonia in Wuhan, China: a single-centered, retrospective, observational study. Lancet Respir Med 2020;8:475-81.

10. Wang Y, Lu X, Li Y, Chen H, Chen T, Su N, et al. Clinical course and outcomes of 344 intensive care patients with COVID-19. Am J Respir Crit Care Med 2020;201:1430-4.

11. Armstrong RA, Kane AD, Cook TM. Outcomes from intensive care in patients with COVID-19: a systematic review and metaanalysis of observational studies. Anaesthesia 2020;75:1340-9.

12. Arentz M, Yim E, Klaff L, Lokhandwala S, Riedo FX, Chong M, et al. Characteristics and outcomes of 21 critically ill patients with COVID-19 in Washington state. JAMA 2020;323:1612-4.

13. Intensive Care National Audit and Research Centre. ICNARC report on COVID-19 in critical care [Internet]. London: Intensive Care National Audit and Research Centre; 2020 [cited 2020 Jul 31]. Available from: https://www.icnarc.org/Our-Audit/Audits/Cmp/Reports.

14. von Baum H, Schweiger B, Welte T, Marre R, Suttorp N, Pletz MW, et al. How deadly is seasonal influenza-associated pneumonia? The German Competence Network for Community-Acquired Pneumonia. Eur Respir J 2011;37:1151-7.

15. Centers for Disease Control and Prevention. Diabetes surveillance system [Internet]. Atlanta (GA): Centers for Disease Control and Prevention [cited 2020 Jul 31]. Available from: https://gis.cdc.gov/grasp/diabetes/DiabetesAtlas.html. 